\title{
Spirituality and Psychological Quality of Life in HIV+ Adults
}

\section{Joshua L. Mc Kelroy and Mark Vosvick, Ph.D.}

Corresponding author contact: Vosvick@unt.edu

Medical advances have lead to the extension of life for many people living with HIV/AIDS (PLH). As a result, Quality of Life (QOL) issues have moved to the forefront of HIV care. This study examined the relationship between spirituality (Ironson-Woods Spirituality/Religiosity Scale, Ironson, 2002) and psychological QOL (MOS-HIV; Wu, 1999). Psychological QOL is a concept which most people have an intuitive

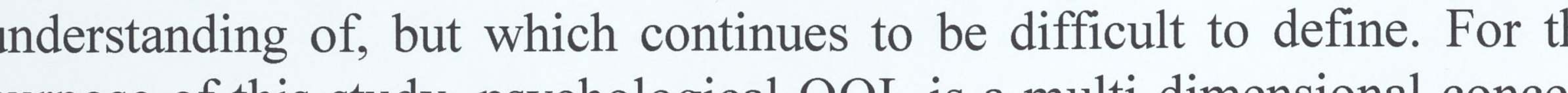
purpose of this study, psychological $\mathrm{QOL}$ is a milti-ding social functioning and energy/fatigue (all scales from the MOS-HIV). We hypothesized that PLH who report higher levels of spirituality would report higher levels of psychological QOL along the five dimension listed. This study included a diverse sample $(\mathrm{N}=245,48 \%$ female; $52.7 \%$ African Americans, $31.8 \%$ European American, $11 \%$ Latino; and $4.5 \%$ other ethnicity, $69.8 \%$ ow incone, age 20.68 . Afrer controlling for were conducted to evaluate factors associated with the total varince in the five psychological QOL dimensions listed. Our findings suggest that som PLH use spiritual beliefs to interpret the meaning of or perhaps apprais illness in such a way that it has a strong positive association with higher levels of psychological QO

Psychological Distress is common in PLH

Mood disorders are associated with

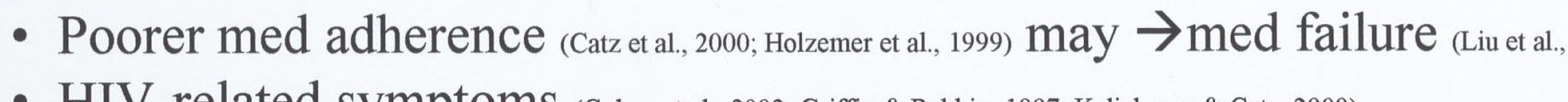

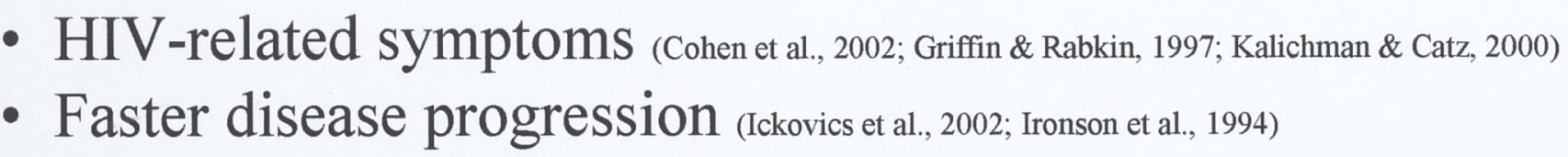

Stressors for PLH are associated with lower QOL

Social related symptoms: finances, lack of HIV-related information, lack of

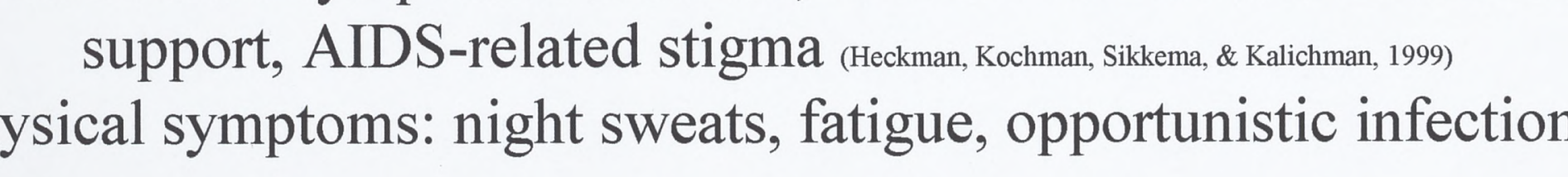

Spirituality coupled in an integral manner with psychological wellbeing may enhance overall QOL

Belief in a higher purpose enables people who are terminally ill to find some

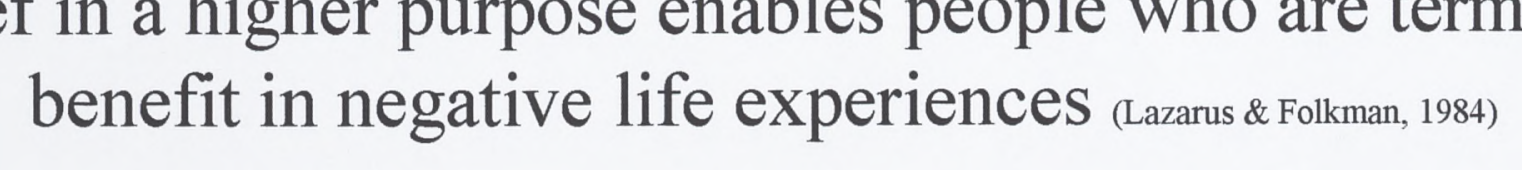

Spirituality is associated with psychological QOL in people living with terminal illness

Hypotheses

People living with HIV/AIDS who report higher levels of life along the five dimensions of cognitive functioning, health
distress, mental health, social functioning, and energy/fatigue.

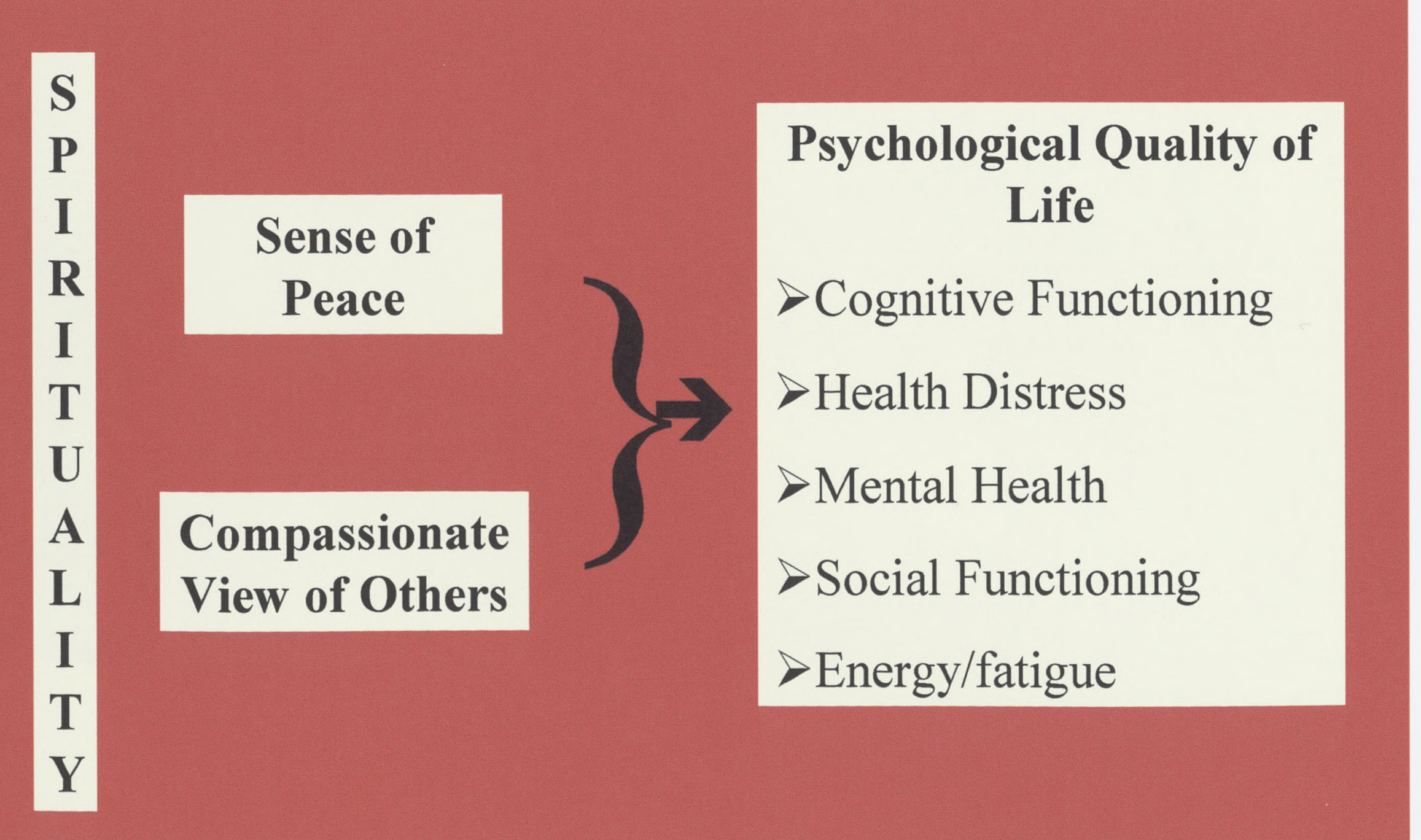

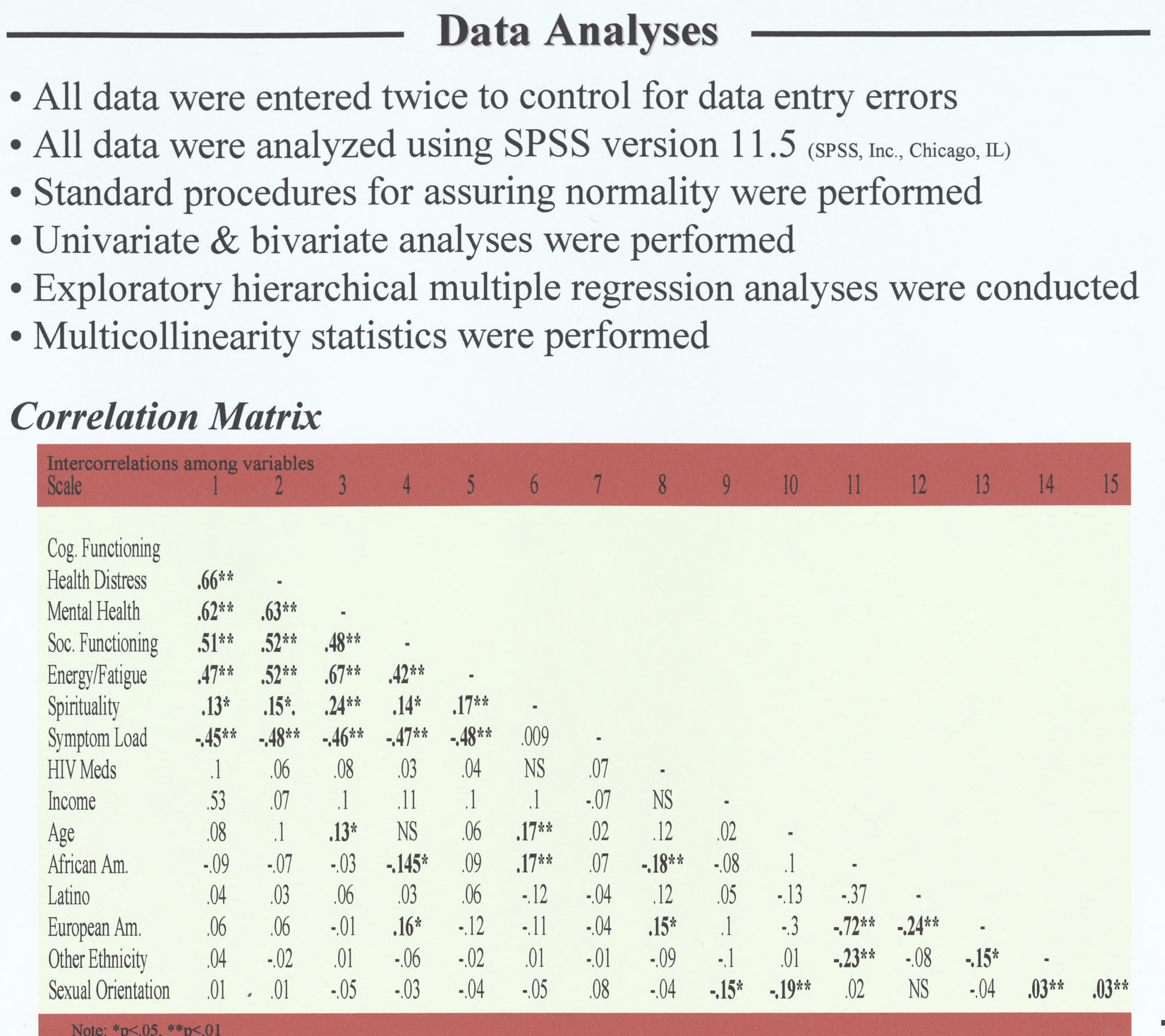

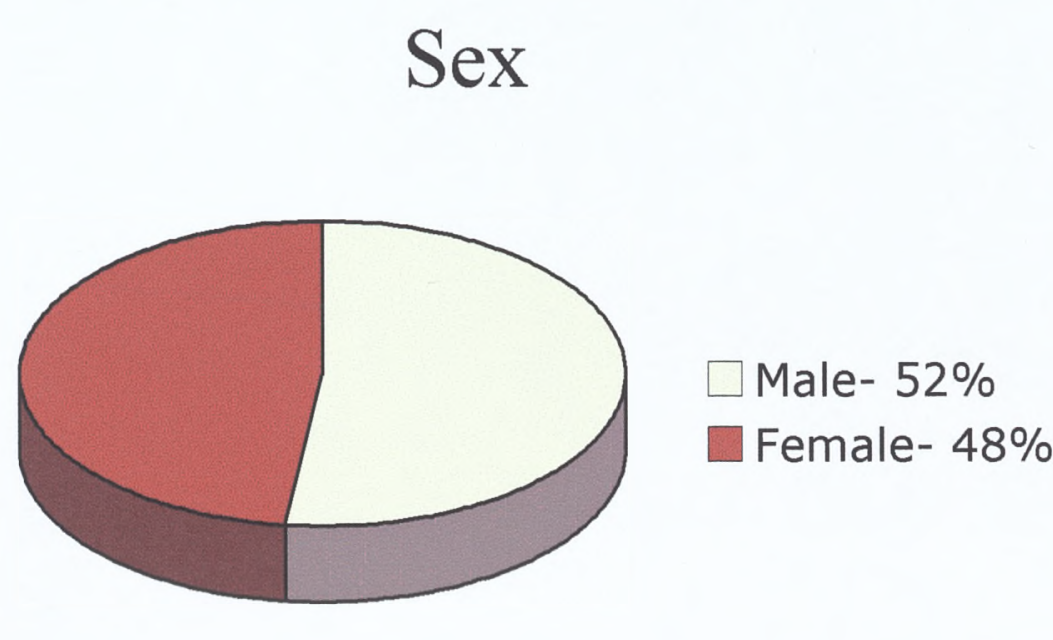

Race/Ethnicity

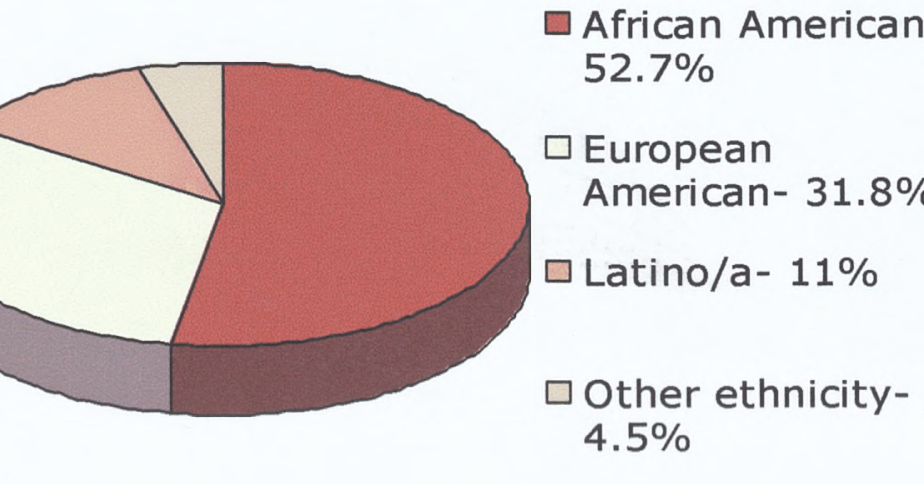

Sexual Orientation

HIV Med Use
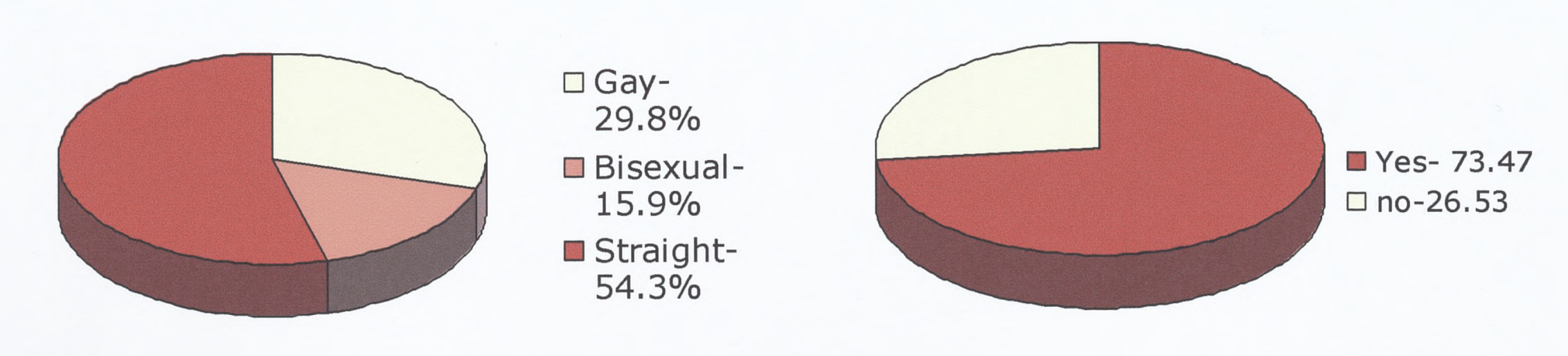

Employmen

Income

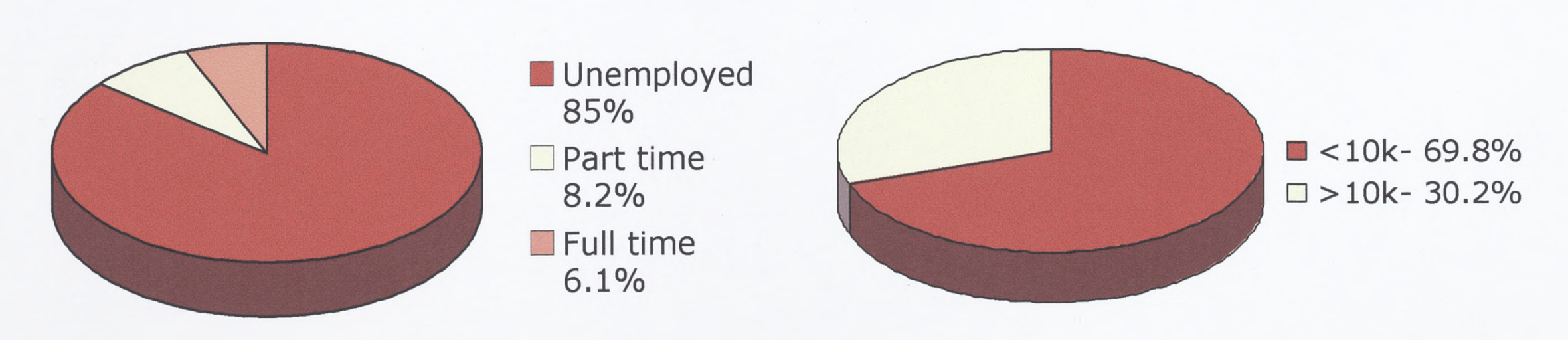

Measures

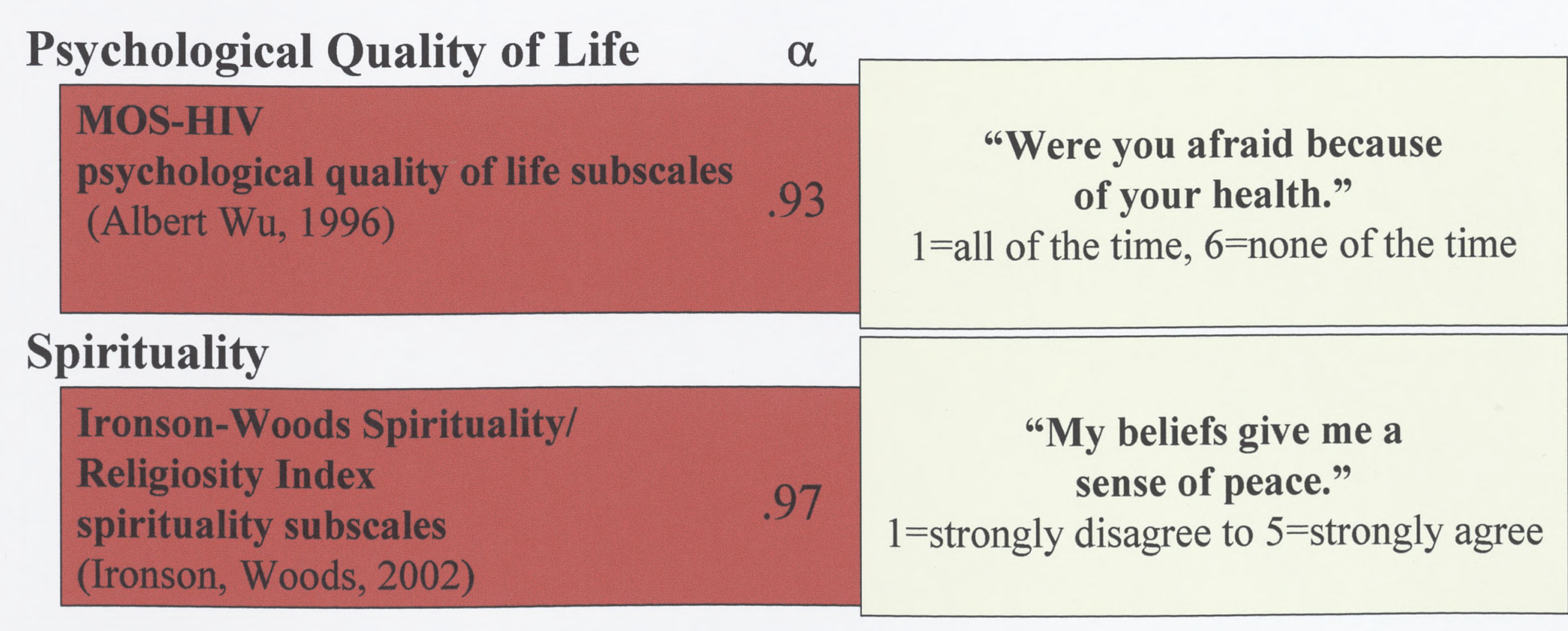

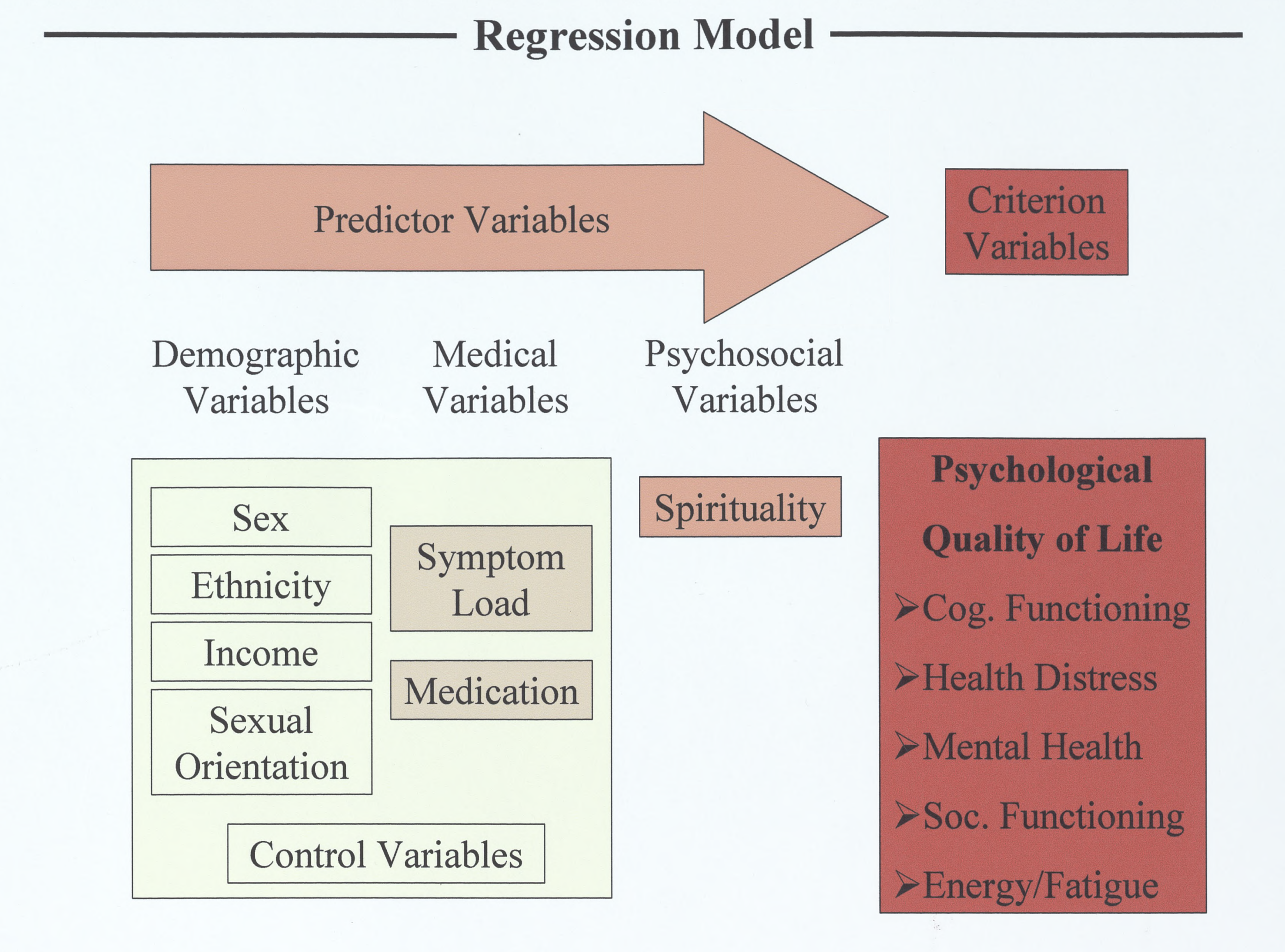

Conclusions

Hypotheses Confirmed - After controlling for demographic and HIV-related varables, higher levels of spirituality were significantly positively associated with higher levels of psychological quality of life along the five dimensions of
cognitive functioning health distress, mental health, social functioning, and cognifive functioning, health distress, mental health, social functioning, and

Clinical Implications

-Clinicians need to be aware that spirituality is strongly associated with sychological quality of life and that, when appropriate, must be addressed during clinician may inadvertently do a disservice to the patient.

-If future studies confirm our hypothesis that the relationship between spirituality and psychological quality of life is a causal one, interventions may target mality of wife as a result of their terminat illess. Treatment interventions weased these at-risk patients may involve raising their awareness of the positive aspects of their life in order to increase their level of spirituality.

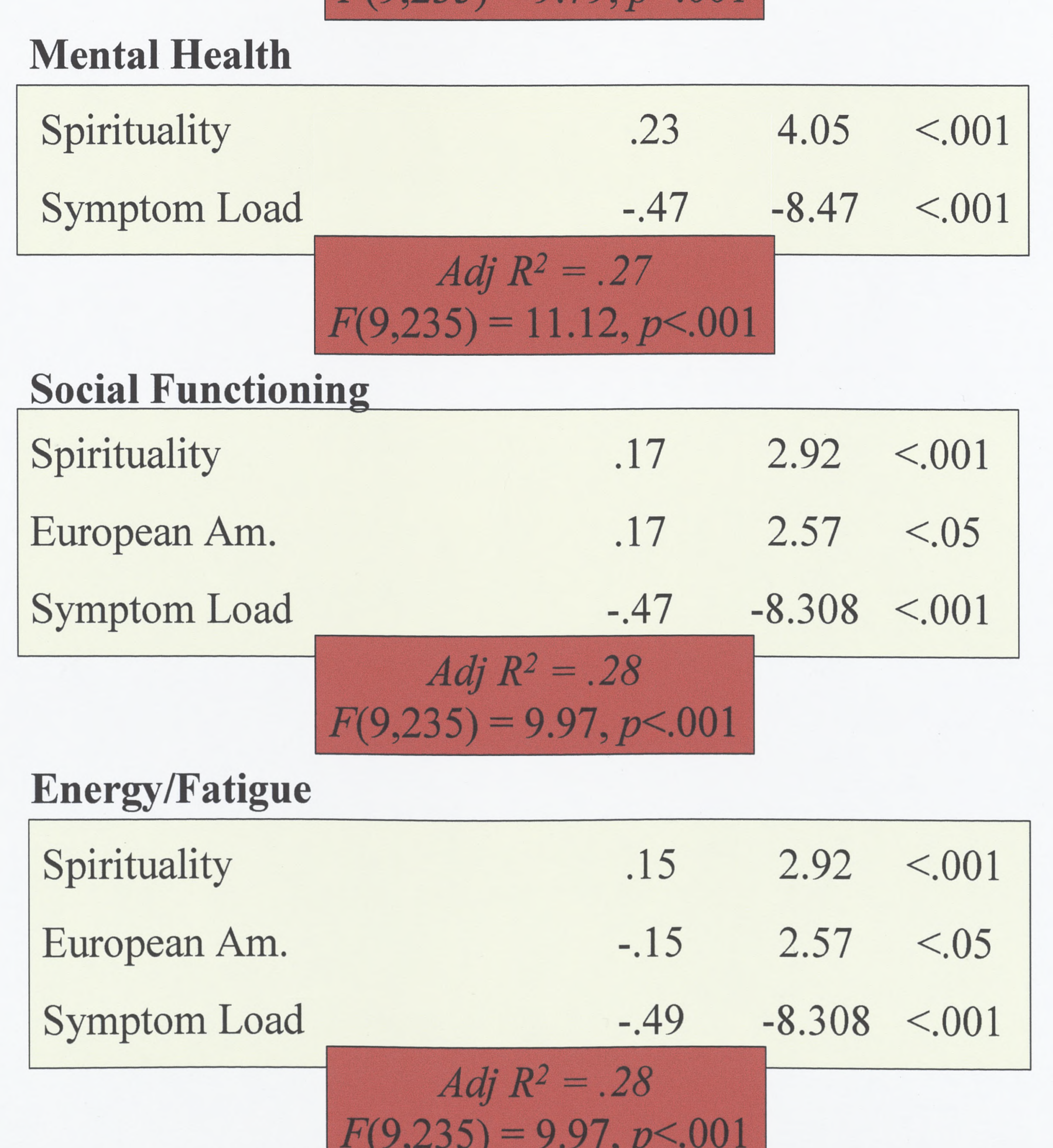

Limitation

-The enrrelational, rross-sectional design of this study limits any causal inferences
Possible unidentified variables could mediate/moderate these relationships. All data was colieeted via self-report measures, which introduced method error variance. Generalizability of our sample may be limited because we used a low-income convenience
sample of PLH in the southern United States who utilize AIDS-service organizations

-Those who have higher income, do not seek services, or live in other regions may differ Future Research

Longitudinal studies (to establish cause and effect relationships) Multiple modes of data collectio Access to middile/high SES PLH

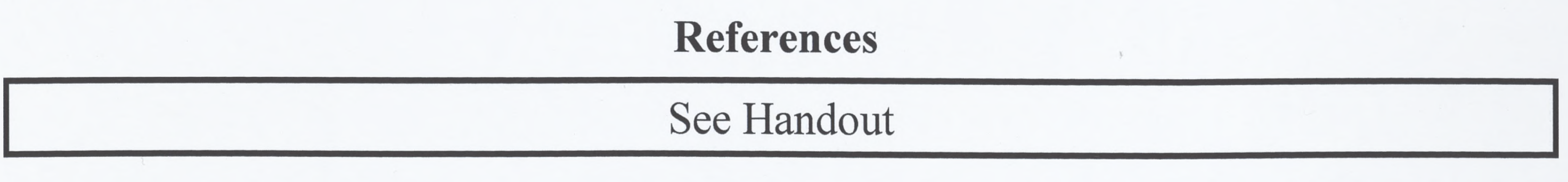

Acknowledgements

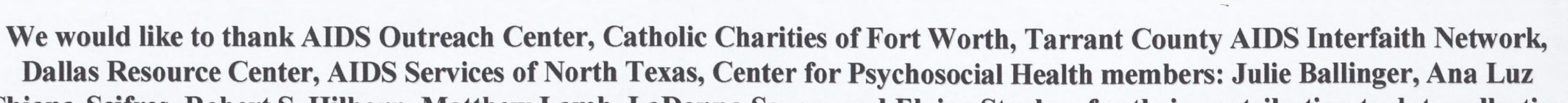

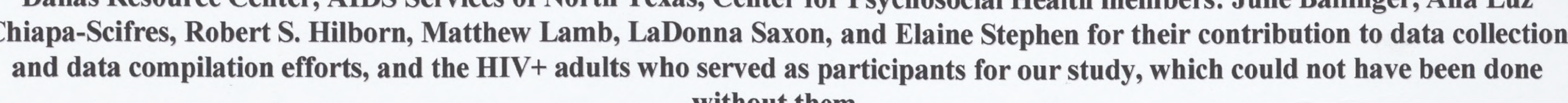


